\title{
Implantable Cardioverter Defibrillator Therapy: A Single Center Experience in Saudi Arabia
}

\author{
Ahmad S. Hersi* \\ Department of Cardiac Sciences, College of Medicine, King Saud University, Riyadh KSA
}

\begin{abstract}
Introduction: Internal Cardioverter Defibrillators (ICD) has been shown to decrease mortality in patients such as those with structural heart disease or at high risk of sudden cardiac death. To date there is no data regarding the clinical features, and outcomes of ICD patients in Saudi Arabia. Accordingly, we explored the clinical features and outcomes of ICD therapy among Saudis.

Methods: Patients who had ICD implantation in King Khalid University Hospital from November 2007 until January 2010 were enrolled.

Results: One hundred and eight ICD were implanted between November 2007 and February 2010. The mean age was $58.6 \pm 13.2$ years. The majority were male 94 (87\%), the rate of Diabetes Mellitus (DM) was 58.3\%, Hypertension (HTN) was 61.1\%, and 63\% were smokers. The mean ejection fraction (EF) was $24.5 \%$. Of the 108 patient 90(83.3\%) had ICD insertion for primary prevention and 18(16.7\%) for secondary prevention. Of the 90 patients who ICD for primary prevention $62(57.4 \%)$ had ischemic cardiomyopathy, 39(36.1\%) had dilated cardiomyopathy and $7(6.5 \%)$ had channelopathy. Over a mean follow-up of 18 months 4 (3.7\%) died
\end{abstract}

Conclusions: Our study describes for the first time patients characteristic and outcomes for ICD therapy in Saudi Arabia. Our patients are younger and have higher prevalence of risk factors that those in Western countries.

Keywords: Implantable Cardioverter-Defibrillator, Saudi Arabia.

\section{INTRODUCTION}

The implantable cardioverter defibrillator (ICD) provides a remarkably effective therapy for ventricular tachycardia (VT) and ventricular fibrillation (VF). Several large, multicenter, randomized clinical trials showed that an ICD is superior to antiarrhythmic drugs in the secondary prevention of sudden cardiac death (SCD) [1-4]. Furthermore, ICD therapy is well-established for primary prevention of SCD, and improves survival in patients with structural heart disease [1,36]. To date, no data are available on characteristics and outcomes of recipients receiving ICD therapy in Saudi Arabia. In the ACC/AHA/HRS 2008 Guidelines for Device-Based Therapy of Cardiac Rhythm Abnormalities, areas in need of further research included identifying and describing "longterm outcomes and risk factors for patients receiving ICDs in general practice compared with trial populations and at academic centers [1]. Accordingly, our objective was to explore clinical features and outcomes of ICD therapy recipients during a 2-year interval in a single academic center in Saudi Arabia.

\section{METHODS}

Patients who had ICD implantation at King Khalid University Hospital in Riyadh, Saudi Arabia, from December

*Address correspondence to this author at the Department of Cardiac Sciences, College of Medicine, King Saud University, Riyadh KSA, Tel: +96614671161; Fax: +9664671158; E-mail: ahersi@ksu.edu.sa
2007 through January 2010 were included in this analysis. Data on patient demographics, clinical history and indication for implantation of the ICD, device type, incidence of shocks, antiarrhythmic medication use, and total mortality were collected. Left ventricular ejection fraction (LVEF) was determined using the Simpson method or by ventriculography during cardiac catheterization. All patients had transvenous ICD insertion via the left axillary vein. None (the electrophysiological study is not anymore a pre-request to ICD insertion as per the guidelines) had an electrophysiological study (EPS) prior to ICD implantation. Patients were required to have Class I indication for ICD therapy as per AHA/ACC/ESC guidelines [1]. In brief, patients qualified for ICD therapy who had: 1) ischemic cardiomyopathy (IC), $\geq 40$ days post myocardial infarction (MI), NYHA functional Class I, and LVEF $\leq 30 \%$; 2) IC, $\geq 40$ days post MI, NYHA Class II or II, and LVEF $\leq 35 \%$; 3 ) dilated cardiomyopathy (DCM) with LVEF $\leq 35 \%$ and NYHA Class II or III; 4) $\geq 40$ days post-MI, LVEF $\leq 40 \%$, and with nonsustained VT and VF or VT inducible at EPS; or 5) cardiac arrest without identifiable reversible cause. After implantation, patients were followed-up regularly in our device clinic. Shocks were documented by device interrogation and confirmed by an electrophysiologist.

Statistical analysis: Continuous variables were summarized as mean \pm standard deviation or median and interquartile range (IQR), as appropriate and categorical variables was summarized as percentages. Group differences between 
categorical variables were analyzed with chi square or Fisher exact test, and between normally distributed continuous variables using Student's t test. For all analyses, $\mathrm{p}<0.05$ was considered significant. Analysis was performed using SPSS version 17.

\section{RESULTS}

From December 2007 through January 2010, 108 ICD implantations were performed. The majority of patients $(\mathrm{n}=$ $94 ; 87 \%$ ) were male, and there was a high prevalence of diabetes mellitus (DM: $\mathrm{n}=63 ; 58.3 \%)$, hypertension $(\mathrm{HTN}: \mathrm{n}=$ $66 ; 61.1 \%)$, dyslipidemia $(\mathrm{n}=51 ; 47.2 \%)$, smoking $(\mathrm{n}=68$; $63 \%)$, and history of previous MI $(\mathrm{n}=57 ; 52.8 \%)$ (Table 1$)$. The mean LVEF was $24.6 \pm 9.43$. The majority were in sinus rhythm $(\mathrm{n}=86 ; 79.6 \%)$, and $22(20.4 \%)$ were in atrial fibrillation (AF). Sixteen (14.8\%) were NYHA Class I, 64 $(59.3 \%)$ were Class II, and 28 (25.9\%) were Class III. Of the 108 implantations, $90(83.3 \%)$ were for primary prevention, and $18(16.7 \%)$ were for secondary prevention. The patients who had ICD therapy for primary prevention were more likely to have ischemic cardiomyopathy $(\mathrm{n}=53 ; 58.9 \%)$ than DCM ( $\mathrm{n}=35 ; 38.9 \%$ : $\mathrm{p}=0.003)$ Table 2 . Of the patients who had ICD for secondary prevention, 7 (38.9\%) had VT with syncope, $8(44.4 \%)$ had cardiac arrest, $2(11.1 \%)$ had long QT syndrome, and $1(5.5 \%)$ had Brugada syndrome. Ninetytwo $(85.2 \%)$ patients had single chamber and $16(14.8 \%)$ had dual chamber ICDs implanted. Over a mean follow-up of $13.4 \pm 9.5$ months, 12 patients $(11.1 \%)$ had ICD shocks, 8 (66.7\% of shocks; $7.4 \%$ of patients) of which were classified as appropriate and 4 (33.3\% of shocks, $3.7 \%$ of patients) as inappropriate. Seven of the appropriate shocks were in patients with VVI-ICD (7.6\% of VVI patients) and one in a patient with DDD-ICD (6.3\% of DDD patients). Appropriate shocks occurred in 5 patients with IC and 3 with DCM. The 4 inappropriate shocks were in patients with VVI-ICD $(4.3 \%$ of VVI patients), 2 of whom had IC and 2 DCM.

All-cause mortality was $3.7 \%(n=4)$; all patients who died had IC, and the indication for implantation was primary prevention, resulting in a $4.4 \%$ mortality rate in that group. The 4 deaths were male, with single chamber ICD, one was in NYHA Class II and 3 were NYHA Class III, 1 was in AF and 3 were in sinus rhythm. None of the deaths occurred in patients experiencing shocks.

\section{DISCUSSION}

Data from multiple clinical trials support the use of ICDs for both primary and secondary prevention of SCD. Several risk factors were used to identify purported high-risk populations to include in those studies [7]. Three large, early RCTs investigating secondary prevention (AVID, CASH, CIDS) enrolled patients who had at least survived VT/VF/cardiac arrest. Although the ICD groups in each study had reduced total mortality, the difference was significant only in the AVID study. The appropriateness of the study risk factors used for eligibility was questioned, however, when a registry of patients not meeting AVID eligibility criteria had worse survival than AVID ICD patients [8] Primary prevention trials had more variable inclusion criteria, and, despite differences in design among the trials, 7 of 9 major trials demonstrated the benefits of ICD therapy [7] The current guidelines on the use of ICDs are based on a writing committee consensus that ICD patients should have clinical profiles closely aligned with those of patients included in those clinical trials; [1] while the controversy over ideal patient characteristics remains, with others reporting that the benefit of ICDs also includes populations not represented in the trials [4].

Table 1. Patient Characteristics and Therapy

\begin{tabular}{|c|c|}
\hline Variable & $\mathrm{n}(\%)$ \\
\hline Age, mean years \pm SD & $58.6 \pm 13.2$ \\
\hline Gender (Male) & $94(87.0)$ \\
\hline $\mathrm{DM}$ & $63(58.3)$ \\
\hline HTN & $66(61.1)$ \\
\hline Dyslipidemia & $51(47.2)$ \\
\hline Stroke & $3(2.8)$ \\
\hline Smoker & $68(63.0)$ \\
\hline MI & $57(52.8)$ \\
\hline $\mathrm{EF}($ mean $\pm \mathrm{SD})$ & $24.6 \pm 9.43$ \\
\hline \multicolumn{2}{|l|}{ Cardiomyopathy: } \\
\hline Ischemic & $62(57.4)$ \\
\hline DCM & $39(36.1)$ \\
\hline Other & $7(6.5)$ \\
\hline \multicolumn{2}{|l|}{ ICD Indication: } \\
\hline Primary & $90(83.3)$ \\
\hline Secondary & $18(16.7)$ \\
\hline \multicolumn{2}{|l|}{ Secondary Prevention: } \\
\hline VT with syncope & $7(38.9)$ \\
\hline Post cardiac arrest & $8(44.4)$ \\
\hline Long QT & $2(11.1)$ \\
\hline Burgada & $1(5.6)$ \\
\hline \multicolumn{2}{|l|}{ Device Type: } \\
\hline VVI - ICD & $92(85.2)$ \\
\hline DDD - ICD & $16(14.8)$ \\
\hline Shocks & $12(11.1)$ \\
\hline Appropriateness & $8(66.6)$ \\
\hline \multicolumn{2}{|l|}{ NYHA: } \\
\hline Class I & $16(14.8)$ \\
\hline Class II & $64(59.3)$ \\
\hline Class III & $28(25.9)$ \\
\hline Class IV & 0 \\
\hline \multicolumn{2}{|l|}{ Rhythm: } \\
\hline SR & $86(79.6)$ \\
\hline $\mathrm{AF}$ & $22(20.4)$ \\
\hline Death & $4(3.7)$ \\
\hline
\end{tabular}


Table 1. contd....

\begin{tabular}{|l|l|}
\hline Variable & \multicolumn{1}{l|}{$\mathbf{n}(\%)$} \\
\hline \hline Medications: & $80(74.1)$ \\
\hline Beta-Blocker & $45(41.7)$ \\
\hline Digoxin & $95(87.9)$ \\
\hline ACE-I & $14(13.0)$ \\
\hline ARB & $98(90.7)$ \\
\hline Statin & $92(85.2)$ \\
\hline Diuretics & $92(85.2)$ \\
\hline Aspirin & $60(55.5)$ \\
\hline Plavix & $10(9.3)$ \\
\hline Warfarin & $23(21.3)$ \\
\hline Nitrate & $39(36.1)$ \\
\hline Spironolactone & $12(11.1)$ \\
\hline Amiodarone & \\
\hline
\end{tabular}

KEY: AF - atrial fibrillation; EF - ejection fraction; ICD - ; SR - sinus rhythm;
LVEF values in the major trials were summarized in a recent systematic review that included 12 efficacy RCTs [4]. Patient LVEF ranged from $21 \%$ to $28 \%$ in primary prevention studies, and $32 \%$ to $46 \%$ in secondary prevention trials. In addition, results from 53 observational effectiveness studies were reviewed, which included patients with mean LVEF ranging from $19 \%$ to $46 \%$. The mean LVEF of $25 \%$ for our patients was within the ranges of the primary prevention RCTs and the observational studies. However, recent studies and commentaries emphasize the importance of identifying risk stratification tools in addition to $\mathrm{EF}$ that can be used in the selection of patients suitable for ICD implantation, and remind that not all patients whose $\mathrm{EF}$ is $\leq 30 \%$ are proper candidates for ICDs [9] In addition, when data from the MUSTT trial were analyzed for the relationship of EF and inducible ventricular tachyarrhythmias with the mode of death, results suggested that ICDs may reduce mortality in patients whose $\mathrm{EF}$ is $\geq 30 \%$ as well as in those with lower EF [10].

Several reports investigated outcomes in patients who met the inclusion criteria of the major studies. For example, a Japanese study retrospectively evaluated data from $90 \mathrm{pa}-$ tients who did not have ICD implantation, but who had the

Table 2. Patients Stratified Based on the Underlying Heart Disease

\begin{tabular}{|c|c|c|c|}
\hline Variable & Ischemic Cardiomyopathy 62(57.4) & DCM 39(36.1) & P-value \\
\hline Age & $60.8 \pm 11.3$ & $57.2 \pm 14.0$ & 0.15 \\
\hline Gender(male) & $59(95.2)$ & $32(82.1)$ & 0.037 \\
\hline DM & $52(57.8)$ & $11(61.1)$ & 0.50 \\
\hline HTN & $41(66.1)$ & $20(51.3)$ & 0.10 \\
\hline Dyslipidemia & $34(54.8)$ & $16(41.0)$ & 0.12 \\
\hline Stroke & $1(1.6)$ & $2(5.1)$ & 0.37 \\
\hline Smoker & $45(72.6)$ & $22(56.4)$ & 0.07 \\
\hline MI & $55(88.7)$ & $2(5.1)$ & $<0.0001$ \\
\hline NYHA Class & & & 0.32 \\
\hline I & $6(9.7)$ & $4(10.3)$ & \\
\hline II & $42(67.7)$ & $21(53.8)$ & \\
\hline III & 13(20.9) & 14(35.9) & \\
\hline $\mathrm{EF}$ & $23.8 \pm 7.5$ & $22.1 \pm 5.3$ & 0.29 \\
\hline \multicolumn{4}{|l|}{ ICD indication } \\
\hline Primary & $53(85.5)$ & $35(89.7)$ & 0.38 \\
\hline Secondary & $9(14.5)$ & $4(10.3)$ & 0.38 \\
\hline Shocks & $7(11.3)$ & $5(12.8)$ & 0.08 \\
\hline Appropriate shocks & $5(8)$ & $3(7.7)$ & 0.3 \\
\hline Death & $4(6.5)$ & $0(.0)$ & 0.12 \\
\hline
\end{tabular}

DM (diabetes mellitus); HTN (hypertension); MI(myocardial infarction); EF(ejection fraction). 
primary prevention RCT MADIT II criteria that included Qwave $\mathrm{MI}>4$ weeks prior, $\mathrm{LVEF}<30 \%$, and age $>21$ years [11]. Survival rates were similar to the ICD group in MADIT II, and greater than the conventional therapy group. However, most patients (79\%) were in NYHA functional Class I, compared with $35 \%$ and $39 \%$ of MADIT-II ICD and conventional therapy patients, respectively [12]. The authors concluded that MADIT-II criteria may be inappropriate for Japanese patients; however, the efficacy of ICDs in Class I patients is not known [1]. In another study, in fact, subgroup analysis of data from 459 patients randomly assigned to standard therapy or standard therapy plus an ICD revealed that LVEF $\geq 20 \%$, male gender, and NYHA Class III but not Class I or II, were significantly associated with improved survival [13]. A Brazilian study compared 102 patients who did not receive an ICD to patients in the placebo arm of another major primary prevention study, SCD-HeFT, and found that the mortality rates were similar between the 2 groups, causing them to conclude that their patients were similar, and may benefit from ICD therapy if economic constraints could be removed [14]. Likewise, our patients shared several similarities with the RCT patients; for example age, gender, proportion of patients with ischemic cardiomyopathy, and distribution among NYHA functional classes was similar to those in the 12 RCTs included in the systematic review by Ezekowitz et al. [4]

The $3.7 \%$ mortality in our entire cohort during a mean follow-up of 13 months is less than that reported in the 11 controlled observational studies included in the systematic review of Ezekowitz et al., where 19 to 1442 patients in each study received an ICD [4]. In follow-up intervals of 12 to 132 months in those studies, all-cause mortality ranged from $6.8 \%$ to $36.8 \%$, with a pooled reduction in all-cause mortality in ICD patients of $46 \%$, which was greater than the $20 \%$ reduction in 9 pooled RCTs [4] All deaths in our study were in patients treated for primary prevention, and the resulting $4.4 \%$ all cause mortality in these patients is less than the $12.3 \%$ to $26.0 \%$ in the 9 primary prevention RCTs in the review. Reduced mortality in ICD patients in observational studies compared with RCTs was suggested by Ezekowitz et al. to indicate that practicing clinicians select healthier patients for ICD insertion [4].

Although ICD effectiveness depends on the device being able to deliver appropriate shock therapy, both therapeutic and inappropriate shocks in patients with ICDs have been shown in several studies to seriously impact quality of life, resulting in decreased physical functioning and mental wellbeing, and increased anxiety that may reach the point of panic disorder or agoraphobia [15]. In fact, in a recent report from China, 1 of 7 deaths following ICD implantation was a suicide response to emotional stress after multiple shocks were received during an electrical storm [16]. Appropriate shocks can be caused by ventricular fibrillation, monomorphic ventricular tachycardia, polymorphic ventricular tachycardia, and Torsades de pointes [15]. From 5\% to $12 \%$ of trial participants received an appropriate shock per year, with reports that it can increase to $30 \%$ of patients by 3 years [4, $17,18]$. In our study, $7.4 \%$ of patients received an appropriate shock, which is much less than the $25 \%$ to $33 \%$ reported in several other observational studies [4]. Brignole [19] emphasizes that "appropriate" and "necessary" shocks are not synonymous, and notes that the low number of patients receiving appropriate shocks was twice the mortality rate in control groups in 2 reports [20].

Rates of inappropriate discharges were reported at 19.1 per 100 patient years in 2 RCTs, and 4.9 per 100 patientyears in 27 observational studies [4] In fact, inappropriate shocks are the most common complication associated with ICD therapy [21]. In one study of 778 patients receiving ICDs, $13 \%$ received an inappropriate shock in follow-up averaging 4 months [22]. These shocks are most commonly due to misdiagnosis of atrial fibrillation or other supraventricular tachycardias, and can also result from double counting, atrial flutter, multiple premature ventricular contractions, oversensing of $\mathrm{T}$ waves or diaphragmatic myopotentials, oversensing due to lead failure or insulation break, electromagnetic interference, and ectopy [23]. Mortality is 2to 5-fold increased in patients who have ICD shocks [17, 19]. This exemplifies the "conversion hypothesis;" that is, patients who are saved from sudden cardiac death by successful shocks may be at high risk of death from other cardiovascular-related causes [24]. Mishkin et al. report that the time from the first ICD shock to death is related to the appropriateness of ICD therapy as well as heart failure etiology, and occurs after a median interval ranging from 168 to 294 days [17].

Our 2-year data revealed that single-chamber devices are primarily used ( $85.2 \%$ of patients) in our institution. Proclemer et al. evaluated data in the Italian Pacemaker Registry and found dual chamber devices in higher use than single chamber devices, with increasing trends toward use of dual chamber devices, similar to trends reported in the world survey [25]. They noted that this was despite a meta-analysis that failed to show a heart failure or overall mortality benefit between the 2 types [26]. In fact, of the 9 RCTs reviewed by Ezekowitcz, only 2 (MADIT-II and COMPANION) evaluated dual chamber devices [4] .Of 20 observational studies of effectiveness that reported the type of device used, 18 included both single- and dual-chamber devices, and one each included only single or dual. Ezekowitcz highlighted the paucity of data on dual chamber devices, remarking that, in addition to 3 observational studies and 1 RCT that failed to show benefit, an RCT suggested that the device can exacerbate heart failure in patients implanted with the dual chamber device without an indication, such as such as chronotropic incompetence, sick sinus syndrome, or atrioventricular conduction abnormalities [27]. There is some evidence to suggest, however, that dual chamber ICDs may be associated with fewer inappropriate shocks [28, 29]. In a recent RCT in which $85 \%$ of patients had indications for secondary prevention, $3 \%$ and $12 \%$ of patients with dual and single-chamber ICDs, respectively, experienced inappropriate shocks in a mean follow-up of 16 months [27]. In our small sample, $4.3 \%$ of patients with single-chamber ICDs had inappropriate shocks. While none of the 16 patients with dual-chamber ICDs had inappropriate shocks, the numbers are too small to allow a valid comparison, which should become possible with the acquisition of additional data on these and other patients. 
This study has several limitations and benefits. In addition to limitations inherent in observational studies, the small numbers reflect that we are just beginning to collect data from a single center. Also, we do not have control group comparisons at this time. However, we are continuing to collect data, and intend to expand participation to include other centers in Saudi Arabia, to acquire data from unimplanted patients for comparison, and to continue follow-up on the patients included in the present report. Our database will be expanded with additional salient variables that will allow more detailed comparisons with data from outside Saudi Arabia, especially those that may be shown to be related to risk factors that could have an impact on patient management and outcome. This report provides the first data from Saudi Arabia on patients implanted with an ICD, establishes a baseline for future comparisons, and launches exploration of the similarities and differences between our patients and those in other populations.

In summary, in our sample of Saudi Arabian patients selected for ICD insertion following practice guidelines, mortality was less than that reported for many RCTs and observational studies [4] suggesting a benefit from ICD insertion in these patients. The importance of acquiring additional data on ICD patients is exemplified by the number of reports questioning patient selection and outcome. For example, in a recent editorial Brignole summarizes ICD efficacy evidence and then questions whether complications may have been underestimated and benefits overestimated [21]. He emphasizes the need to develop reliable tools to identify patients who should receive ICD therapy. Real world data such as ours provide additional information outside of clinical trials, and ongoing surveillance and evaluation of outcomes and relevance of prediction models can help guide management of these patients. Our work is in line with the conclusion of the Guideline Committee that an area in need of further research is determining long-term outcomes and risk factors for patients receiving ICDs in general practice, and comparing them with trial populations and at academic centers. The acquisition of additional data will allow more meaningful comparisons between our data and that obtained in RCTs and observational studies in other populations.

\section{REFERENCES}

[1] Epstein AE, DiMarco JP, Ellenbogen KA, et al. ACC/AHA/HRS 2008 Guidelines for Device-Based Therapy of Cardiac Rhythm Abnormalities. J Am Coll Cardiol 2008; 51: 1-62.

[2] Connolly SJ, Hallstrom AP, Cappato R, et al. Meta-analysis of the implantable cardioverter defibrillator secondary prevention trials. AVID, CASH and CIDS studies. Antiarrhythmics vs Implantable Defibrillator study. Cardiac Arrest Study Hamburg. Canadian Implantable Defibrillator Study. Eur Heart J 2000; 21: 2071- 8.

[3] Ezekowitz JA, Armstrong PW, McAlister FA. Implantable cardioverter defibrillators in primary and secondary prevention: a systematic review of randomized, controlled trials. Ann Intern Med 2003; 138: 445-52.

[4] Ezekowitz JA, Rowe BH, Dryden DM, et al. Systematic review: implantable cardioverter defibrillators for adults with left ventricular systolic dysfunction. Ann Intern Med 2007; 147: 25162.

[5] Zwanziger J, Hall WJ, Dick AW, et al. The cost effectiveness of implantable cardioverter-defibrillators: results from the Multicenter Automatic Defibrillator Implantation Trial (MADIT)-II. J Am Coll Cardiol 2006; 47: 2310-8.
[6] Mark DB, Nelson CL, Anstrom KJ, et al. Cost-effectiveness of defibrillator therapy or amiodarone in chronic stable heart failure: results from the Sudden Cardiac Death in Heart Failure Trial (SCDHeFT). Circulation 2006; 114: 135-42.

[7] Goldberger Z, Lampert R. Implantable cardioverter-defibrillators: expanding indications and technologies. JAMA 2006; 295: 809818.

[8] Anderson JL, Hallstrom AP, Epstein AE, et al. AVID Investigators. Design and results of the Antiarrhythmics vs Implantable Defibrillators (AVID) registry. Circulation1999; 99:1692-9.

[9] Buxton AE, Moss AJ. Should everyone with an ejection fraction less than or equal to $30 \%$ receive an implantable cardioverterdefibrillator? Circulation 2005; 111: 2537-49.

[10] Buxton AE, Lee KL, Hafley GE, et al. Relation of ejection fraction and inducible ventricular tachycardia to mode of death in patients with coronary artery disease: An analysis of patients enrolled in the multicenter unsustained tachycardia trial. Circulation 2002; 106; 2466-72;

[11] Tanno K, Miyoshi F, Watanabe N, et al. Are the MADIT II criteria for ICD implantation appropriate for Japanese patients? Circ J 2005; 69: 19-22.

[12] Moss AJ, Zareba W, Hall WJ, et al. Prophylactic implantation of a defibrillator in patients with myocardial infarction and reduced ejection fraction. N Engl J Med 2002; 346: 877-83.

[13] Kadish A, Dyer A, Daubert JP, et al. For the defibrillators in nonischemic cardiomyopathy treatment evaluation (DEFINITE) investigators. N Engl J Med 2004; 350: 2151-8.

[14] Hadid C, Avellana P, Di Toro D, Gomez CF, Visser M, Prieto N. Long-term follow-up of patients with indication for a implantable defibrillator for primary prevention of death. Arq Bras Cardiol 2008; 90: 311-5.

[15] Gehi AK, Mehta D, Gomes JA. Evaluation and management of patients after implantable cardioverter-defibrillator shock. JAMA 2006; 296: 2839-47.

[16] Chen T-E, Wang C-C, Chang S-H, Yeh S-J, Wu D. Implantable cardioverter defibrillator therapy: ten years experience in a medical center. Chang Gung Med J 2008; 31: 81-90

[17] Mishkin JD, Saxonhouse SJ, Woo GW, et al. Appropriate evaluation and treatment of heart failure patients after implantable cardioverter-defibrillator discharge. J Am Coll Cardiol 2009; 54: 1993-2000.

[18] Stevenson, LW. Implantable Cardioverter-Defibrillators for Primary Prevention of Sudden Death in Heart Failure: Are There Enough Bangs for the Bucks? Circulation 2006; 114: 101-3.

[19] Brignole M. Are complications of implantable defibrillators underestimated and benefits over-estimated? Europace 2009; 11: 112933.

[20] Tung R, Swerdlow CD. Refining patient selection for primary prevention implantable cardioverter-defibrillator therapy: reeling in a net cast too widely. Circulation 2009; 120: 825-827.

[21] Duray GZ, Schmitt J, Cicek-Hartvig S, Hohnloser SH, Israel CW. Complications leading to surgical revision in implantable cardioverter defibrillator patients: comparison of patients with singlechamber, dual-chamber, and biventricular devices. Europace 2009; 11: 297-302.

[22] Rosenqvist M, Beyer T, Block M, den Dulk K, Minten J, Lindemans F; European and the 7219 Jewel ICD Investigators. Adverse events with transvenous implantable cardioverter-defibrillators: a prospective multicenter study. Circulation 1998; 98: 663-70.

[23] Dorian P. Effective and efficient use of implantable defibrillators: sometimes it's over when it's over. CMAJ 2009; 180: 599-600.

[24] Proclemer A, Ghidina M, Gregori D, et al. Trend of the main clinical characteristics and pacing modality in patients treated by pacemaker: data from the Italian Pacemaker Registry for the quinquennium 2003-07. Europace 2010; 12: 202-9.

[25] Healey J, Toff W, Lamas G, et al. Cardiovascular outcomes with atrial-based pacing compared with ventricular pacing: meta-analysis of randomized trials, using individual patient data. Circulation 2006; 114: 11-17.

[26] The DAVID Trial Investigators. Dual-chamber pacing or ventricular backup pacing in patients with an implantable defibrillator: the Dual Chamber and VVI Implantable Defibrillator (DAVID) trial. JAMA 2002; 288: 3115-23.

[27] Bansch D, Steffgen F, Gronefeld G, et al. The f 11 trial: a prospective trial of a dual- versus a single-chamber implantable defibrilla- 
tor in patients with slow ventricular tachycardias. Circulation 2004; 110: 1022-9.

[28] Friedman PA, McClelland RL, Bamlet WR, et al. Dual-chamber versus single-chamber detection enhancements for implantable defibrillator rhythm diagnosis. The Detect Supraventricular Tachycardia Study. Circulation 2006; 113: 28719.
[29] Almendral J, Arribas F, Wolpert C, et al, and the DATAS Steering Committee and Writing Committee on behalf of the DATAS Investigators. Dual-chamber defibrillators reduce clinically significant adverse events compared with single-chamber devices: results from the DATAS (Dual chamber and Atrial Tachyarrhythmias Adverse events Study) trial. Europace 2008; 10: 528-35.

(C) Ahmad S. Hersi; Licensee Bentham Open.

This is an open access article licensed under the terms of the Creative Commons Attribution Non-Commercial License (http://creativecommons.org/licenses/ by-nc/3.0/) which permits unrestricted, non-commercial use, distribution and reproduction in any medium, provided the work is properly cited. 